\title{
Bifurcation from Interval and Positive Solutions of a Nonlinear Second-Order Dynamic Boundary Value Problem on Time Scales
}

\author{
Hua Luo \\ School of Mathematics and Quantitative Economics, Dongbei University of Finance and Economics, \\ Dalian 116025, China \\ Correspondence should be addressed to Hua Luo, luohuanwnu@gmail.com \\ Received 25 September 2012; Accepted 9 November 2012 \\ Academic Editor: Paul Eloe \\ Copyright (c) 2012 Hua Luo. This is an open access article distributed under the Creative Commons \\ Attribution License, which permits unrestricted use, distribution, and reproduction in any \\ medium, provided the original work is properly cited. \\ Let $\mathbb{T}$ be a time scale with $0, T \in \mathbb{T}$. We give a global description of the branches of positive solutions \\ to the nonlinear boundary value problem of second-order dynamic equation on a time scale $\mathbb{T}$, \\ $u^{\Delta \Delta}(t)+f\left(t, u^{\sigma}(t)\right)=0, t \in[0, T]_{\mathbb{T}}, u(0)=u\left(\sigma^{2}(T)\right)=0$, which is not necessarily linearizable. Our \\ approaches are based on topological degree theory and global bifurcation techniques.
}

\section{Introduction}

Let $\mathbb{T}$ be a time scale with $0, T \in \mathbb{T}$, we consider the existence of positive solutions, in this paper, for a nonlinear boundary value problem of second-order dynamic equation on a time scale $\mathbb{T}$ as follows:

$$
\begin{gathered}
u^{\Delta \Delta}(t)+f\left(t, u^{\sigma}(t)\right)=0, \quad t \in[0, T]_{\mathbb{T}} \\
u(0)=u\left(\sigma^{2}(T)\right)=0 .
\end{gathered}
$$

Research for the existence of solutions to the dynamic boundary value problem is rapidly growing in recent years. A great many existence results of positive solutions have been established for problem (1.1), see [1-5] and the references therein. The main tool used by 
them is the fixed point theorem in cones, and the key conditions in these papers do not depend on the first eigenvalue, $\lambda_{1}(a)$, of the following linear problem:

$$
\begin{gathered}
u^{\Delta \Delta}(t)+\lambda a(t) u^{\sigma}(t)=0, \quad t \in[0, T]_{\mathbb{T}} \\
u(0)=u\left(\sigma^{2}(T)\right)=0
\end{gathered}
$$

and the corresponding existence conditions are not optimal.

In 2006, for $0,1 \in \mathbb{T}, \sigma(0)=0, \rho(1)=1, \widehat{f} \in C(\mathbb{R})$ with $s \widehat{f}(s)>0$ for $s \neq 0$, Luo and Ma [6] obtained the existence of at least one positive solution to problem:

$$
\begin{gathered}
u^{\Delta \Delta}(t)+\widehat{f}\left(u^{\sigma}(t)\right)=0, \quad t \in[0,1]_{\mathbb{T}}, \\
u(0)=u(1)=0,
\end{gathered}
$$

under the condition

(H) if either $\widehat{f}_{0}<\mu_{1}<\widehat{f}_{\infty}$ or $\widehat{f}_{\infty}<\mu_{1}<\widehat{f}_{0}$, where

$$
\widehat{f}_{0}=\lim _{|s| \rightarrow 0} \frac{\widehat{f}(s)}{s}, \quad \widehat{f}_{\infty}=\lim _{|s| \rightarrow \infty} \frac{\widehat{f}(s)}{s}
$$

and $\mu_{1}$ is the first eigenvalue of the linear problem:

$$
u^{\Delta \Delta}(t)+\mu u^{\sigma}(t)=0, \quad u(0)=u(1)=0 .
$$

The approaches adopted by Luo and Ma [6] are based on global bifurcation techniques. They obtained the existence of at least one positive solution by considering the branches of solutions, which bifurcate from one point. The key conditions in [6] depend on the first eigenvalue of the corresponding linear problem and the condition $(\mathrm{H})$ is optimal!

In this paper, we will use the following assumptions.

(A1) $f:[0, \sigma(T)]_{\mathbb{T}} \times[0, \infty) \rightarrow[0, \infty)$ is continuous and there exist functions $a_{0}(\cdot), a^{0}(\cdot), b_{\infty}(\cdot), b^{\infty}(\cdot) \in C\left([0, \sigma(T)]_{\mathbb{T}},(0, \infty)\right)$, such that

$$
a_{0}(t) u-\xi_{1}(t, u) \leq f(t, u) \leq a^{0}(t) u+\xi_{2}(t, u)
$$

for some functions $\xi_{i} \in C\left([0, \sigma(T)]_{\mathbb{T}} \times[0, \infty), \mathbb{R}\right)$ with $\lim _{u \rightarrow 0^{+}}\left(\xi_{i}(t, u) / u\right)=0(i=$ $1,2)$ uniformly for $t \in[0, \sigma(T)]_{\mathbb{T}}$, and

$$
b_{\infty}(t) u-\zeta_{1}(t, u) \leq f(t, u) \leq b^{\infty}(t) u+\zeta_{2}(t, u)
$$

for some functions $\zeta_{i} \in C\left([0, \sigma(T)]_{\mathbb{T}} \times[0, \infty), \mathbb{R}\right)$ with $\lim _{u \rightarrow+\infty}\left(\zeta_{i}(t, u) / u\right)=0(i=$ $1,2)$ uniformly for $t \in[0, \sigma(T)]_{\mathbb{T}}$. 
(A2) $f(t, u)>0$ for $(t, u) \in[0, \sigma(T)]_{\mathbb{T}} \times(0, \infty)$.

(A3) There exists a function $c \in C\left([0, \sigma(T)]_{\mathbb{T}},(0, \infty)\right)$ such that

$$
f(t, u) \geq c(t) u, \quad(t, u) \in[0, \sigma(T)]_{\mathbb{T}} \times[0, \infty) .
$$

Obviously, (A1) means that $f$ is not necessarily linearizable at 0 and $+\infty$. We consider the existence of positive solutions of problem (1.1) in this paper by using bifurcation techniques. The difference from [6] is that the branches of positive solutions under consideration now bifurcate from not one point, but an interval. Our main idea is from [7], in which they considered positive solutions of fourth-order boundary value problems for differential equations. The main tool we will use is the following global bifurcation theorem for problems which is not necessarily linearizable.

Theorem A (Rabinowitz, [8]). Let $V$ be a real reflexive Banach space. Let $F: \mathbb{R} \times V \rightarrow V$ be completely continuous such that $F(\lambda, 0)=0$, for all $\lambda \in \mathbb{R}$. Let $a, b \in \mathbb{R}(a<b)$ be such that $u=0$ is an isolated solution of the equation

$$
u-F(\lambda, u)=0, \quad u \in V,
$$

for $\lambda=a$, and $\lambda=b$, where $(a, 0),(b, 0)$ are not bifurcation points of (1.9). Furthermore, assuming that

$$
\operatorname{deg}\left(I-F(a, \cdot), B_{r}(0), 0\right) \neq \operatorname{deg}\left(I-F(b, \cdot), B_{r}(0), 0\right),
$$

where $B_{r}(0)$ is an isolating neighborhood of the nontrivial solution, and $\operatorname{deg}\left(I-F, B_{r}(0), 0\right)$ denote the degree of $I-F$ on $B_{r}(0)$ with respect to 0 . Let

$$
\mathcal{S}=\overline{\{(\lambda, u):(\lambda, u) \text { is a solution of }(1.9) \text { with } u \neq 0\}} \cup([a, b] \times\{0\}) .
$$

Then there exists a connected component $\mathcal{C}$ of $\mathcal{S}$ containing $[a, b] \times\{0\}$, and either

(i) $C$ is unbounded, or

(ii) $\mathcal{C} \cap[(\mathbb{R} \backslash[a, b]) \times\{0\}] \neq \emptyset$.

The rest of the paper is organized as follows. In Section 2, we firstly introduce the time scales concepts and notations that we will use in this paper. Next, Section 3 states some notations and proves some necessary preliminary results, and Section 4 studies the bifurcation from the trivial solution for a nonlinear problem which is not necessarily linearizable and then establishes our main result. 


\section{Introduction for Time Scales}

A time scale $\mathbb{T}$ is a nonempty closed subset of $\mathbb{R}$, assuming that $\mathbb{T}$ has the topology that it inherits from the standard topology on $\mathbb{R}$. Define the forward and backward jump operators $\sigma, \rho: \mathbb{T} \rightarrow \mathbb{T}$ by

$$
\sigma(t)=\inf \{\tau>t \mid \tau \in \mathbb{T}\}, \quad \rho(t)=\sup \{\tau<t \mid \tau \in \mathbb{T}\}
$$

Here, we put $\inf \emptyset=\sup \mathbb{T}$, $\sup \emptyset=\inf \mathbb{T}$. Let $\mathbb{T}^{k}$ which is derived from the time scale $\mathbb{T}$ be

$$
\mathbb{T}^{k}:=\{t \in \mathbb{T}: t \text { is nonmaximal or } \rho(t)=t\},
$$

and $\mathbb{T}^{k^{2}}:=\mathbb{T}^{k}$. Define interval $I$ on $\mathbb{T}$ by $I_{\mathbb{T}}=I \cap \mathbb{T}$.

Definition 2.1. If $u: \mathbb{T} \rightarrow \mathbb{R}$ is a function and $t \in \mathbb{T}^{k}$, then the $\Delta$-derivative of $u$ at the point $t$ is defined to be the number $u^{\Delta}(t)$ (provided that it exists) with the property that for each $\varepsilon>0$, there is a neighborhood $U$ of $t$ such that

$$
\left|u(\sigma(t))-u(s)-u^{\Delta}(t)(\sigma(t)-s)\right| \leqslant \varepsilon|\sigma(t)-s|
$$

for all $s \in U$. The function $u$ is called $\Delta$-differentiable on $\mathbb{T}$ if $u^{\Delta}(t)$ exists for all $t \in \mathbb{T}^{k}$.

The second $\Delta$-derivative of $u$ at $t \in \mathbb{T}^{k^{2}}$, if it exists, is defined to be $u^{\Delta^{2}}(t)=u^{\Delta \Delta}(t):=$ $\left(u^{\Delta}\right)^{\Delta}(t)$. We also define the function $u^{\sigma}:=u \circ \sigma$ and $u^{\rho}:=u \circ \rho$.

Definition 2.2. If $U^{\Delta}=u$ holds on $\mathbb{T}^{k}$, we define the Cauchy $\Delta$-integral by

$$
\int_{s}^{t} u(\tau) \Delta \tau=U(t)-U(s), \quad s, t \in \mathbb{T}^{k}
$$

Lemma 2.3 (see [2, Theorems 2.7 and 2.8]). Assume $a, b \in \mathbb{T}$, then

$$
\int_{a}^{b} f^{\Delta}(t) g(t) \Delta t=\left.f(t) g(t)\right|_{a} ^{b}-\int_{a}^{b} f^{\sigma}(t) g^{\Delta}(t) \Delta t
$$

Furthermore, if $a \leq b, f(t)$ is a continuous function on $[a, b]$, then

$$
\int_{a}^{b} f(t) \Delta t=\int_{a}^{\rho(b)} f(t) \Delta t+[b-\rho(b)] f(\rho(b)) .
$$

Define the Banach space $C(\mathbb{T}, \mathbb{R})$ (denoted by $C(\mathbb{T})$ ) to be the set of continuous functions $u: \mathbb{T} \rightarrow \mathbb{R}$ with the norm

$$
\|u\|_{0}=\sup \{|u(t)| \mid t \in \mathbb{T}\}
$$


For $i=1,2$, we define the Banach space $C^{i}(\mathbb{T})$ to be the set of the $i$ th $\Delta$-differential functions $u: \mathbb{T} \rightarrow \mathbb{R}$ for which $u^{\Delta^{i}} \in \mathrm{C}\left(\mathbb{T}^{k^{i}}\right)$ with the norm

$$
\|u\|_{i}=\sup \left\{\|u\|_{\infty},\left\|u^{\Delta}\right\|_{0^{\prime}}, \ldots,\left\|u^{\Delta^{i}}\right\|_{0}\right\}
$$

where

$$
\left\|u^{\Delta^{j}}\right\|_{0}=\sup \left\{\left|u^{\Delta^{j}}(t)\right| \mid t \in \mathbb{T}^{k^{j}}\right\}, \quad j=0,1, \ldots, i
$$

\section{Preliminaries and Necessary Lemmas}

Assuming that $a \in \mathrm{C}\left(\left[0, \sigma^{2}(T)\right]_{\mathbb{T}},(0, \infty)\right)$, then from $[9$, Theorem 2.9], linear problem

$$
\begin{gathered}
u^{\Delta \Delta}(t)+\lambda a(t) u^{\sigma}(t)=0, \quad t \in[0, T]_{\mathbb{T}} \\
u(0)=u\left(\sigma^{2}(T)\right)=0
\end{gathered}
$$

has a unique principal eigenvalue $\lambda_{1}(a)$, with a corresponding positive eigenfunction.

Let $E=C^{2}[0, T]_{\mathbb{T}}, X=C[0, T]_{\mathbb{T}}$, and

$$
Y=\left\{y \in C^{1}\left[0, \sigma^{2}(T)\right]_{\mathbb{T}} \mid y(0)=y\left(\sigma^{2}(T)\right)=0\right\} .
$$

We will work essentially in the Banach space $Y$ with the norm

$$
\|y\|=\max \left\{\|y\|_{0},\left\|y^{\Delta}\right\|_{0}\right\}
$$

where

$$
\|y\|_{0}=\sup \left\{|y(t)| \mid t \in\left[0, \sigma^{2}(T)\right]_{\mathbb{T}}\right\}, \quad\left\|y^{\Delta}\right\|_{0}=\sup \left\{\left|y^{\Delta}(t)\right| \mid t \in[0, \sigma(T)]_{\mathbb{T}}\right\} .
$$

By a positive solution of problem (1.1), we mean $u$ is a solution of $(1.1)$ with $u \geq 0$ in $\left(0, \sigma^{2}(T)\right)_{\mathbb{T}}$ and $u \neq \equiv$.

Lemma 3.1. For $y \in Y$, we have

$$
\|y\|_{0} \leq \sigma(T)\left\|y^{\Delta}\right\|_{0^{\prime}} \quad \max \left\{1, \frac{1}{\sigma(T)}\right\}\|y\|_{0} \leq\|y\| \leq \max \{1, \sigma(T)\}\left\|y^{\Delta}\right\|_{0} .
$$

Proof. By $y(0)=0$, we have that

$$
y(t)=\int_{0}^{t} y^{\Delta}(s) \Delta s, \quad t \in[0, \sigma(T)]_{\mathbb{T}}
$$


and so

$$
\|y\|_{0} \leq \sigma(T)\left\|y^{\Delta}\right\|_{0}
$$

Therefore,

$$
\begin{gathered}
\|y\|=\max \left\{\|y\|_{0},\left\|y^{\Delta}\right\|_{0}\right\} \leq \max \{1, \sigma(T)\}\left\|y^{\Delta}\right\|_{0^{\prime}} \\
\|y\| \geq \max \left\{\|y\|_{0}, \frac{1}{\sigma(T)}\|y\|_{0}\right\}=\max \left\{1, \frac{1}{\sigma(T)}\right\}\|y\|_{0} .
\end{gathered}
$$

Define the linear operator $L: D(L) \subset E \rightarrow X$,

$$
L u=-u^{\Delta \Delta}, \quad u \in D(L)
$$

with

$$
D(L)=\left\{u \in E \mid u(0)=u\left(\sigma^{2}(T)\right)=0\right\} .
$$

Then $L$ is a closed operator, and $L^{-1}: X \rightarrow Y$ is completely continuous, see [10, Lemma 3.7]. Let $\Sigma \subset \mathbb{R}^{+} \times Y$ be the closure of the set of positive solutions to the problem

$$
L u(t)=\lambda f\left(t, u^{\sigma}(t)\right), \quad t \in[0, \sigma(T)]_{\mathbb{T}}
$$

We extend the function $f$ to a continuous function $\bar{f}$ defined on $[0, \sigma(T)]_{\mathbb{T}} \times \mathbb{R}$ by

$$
\bar{f}(t, u)= \begin{cases}f(t, u), & (t, u) \in[0, \sigma(T)]_{\mathbb{T}} \times[0, \infty) \\ f(t, 0), & (t, u) \in[0, \sigma(T)]_{\mathbb{T}} \times(-\infty, 0)\end{cases}
$$

Then $\bar{f}(t, u) \geq 0$ on $[0, \sigma(T)]_{\mathbb{T}} \times \mathbb{R}$. For $\lambda \geq 0$, the arbitrary solution $u$ to the eigenvalue problem

$$
\begin{gathered}
u^{\Delta \Delta}(t)+\lambda \bar{f}\left(t, u^{\sigma}(t)\right)=0, \quad t \in[0, T]_{\mathbb{T}}, \\
u(0)=u\left(\sigma^{2}(T)\right)=0
\end{gathered}
$$

satisfies that $u^{\Delta \Delta}(t) \leq 0$ on $[0, T]_{\mathbb{T}}$, and consequently, the graph of $u$ is concave down on $\left[0, \sigma^{2}(T)\right]_{\mathbb{T}}$. This together with the boundary conditions $u(0)=u\left(\sigma^{2}(T)\right)=0$ imply that

$$
u(t) \geq 0, \quad t \in\left[0, \sigma^{2}(T)\right]_{\mathbb{T}} .
$$

Thus, (3.14) implies that $u$ is a nonnegative solution of problem (3.13), and the closure of the set of nontrivial solutions $(\lambda, u)$ of $(3.13)$ in $\mathbb{R}^{+} \times Y$ is exactly $\Sigma$. 
Let $g \in \mathrm{C}\left([0, \sigma(T)]_{\mathbb{T}} \times \mathbb{R}, \mathbb{R}\right)$, and let $\widehat{N}: Y \rightarrow X$ be the Nemytskii operator associated with the function $g$ :

$$
\widehat{N}(u)(t)=g\left(t, u^{\sigma}(t)\right), \quad u \in Y .
$$

Lemma 3.2. Let $g(t, u) \geq 0$ on $[0, \sigma(T)]_{\mathbb{T}} \times \mathbb{R}$. Let $u \in D(L)$ be such that $L u \geq \lambda \widehat{N}(u)$ in $[0, T]_{\mathbb{T}}$, $\lambda \geq 0$. Then

$$
u(t) \geq 0, \quad t \in\left[0, \sigma^{2}(T)\right]_{\mathbb{T}} .
$$

Moreover,

$$
u(t)>0, \quad t \in\left(0, \sigma^{2}(T)\right)_{\mathbb{T}^{\prime}}
$$

whenever $u \neq \equiv$.

Let $N: Y \rightarrow X$ be the Nemytskii operator associated with the function $\bar{f}$

$$
N(u)(t)=\bar{f}\left(t, u^{\sigma}(t)\right), \quad u \in Y .
$$

Then (3.13), with $\lambda \geq 0$, is equivalent to the operator equation

$$
u=\lambda L^{-1} N(u), \quad u \in Y .
$$

In the following we will apply the Brouwer degree theory, mainly to the mapping $\Phi_{\curlywedge}: Y \rightarrow$ Y,

$$
\Phi_{\curlywedge}(u)=u-\lambda L^{-1} N(u)
$$

For $R>0$, let $B_{R}=\{u \in Y:\|u\|<R\}$.

Lemma 3.3. Let $\Lambda \subset \mathbb{R}^{+}$be a compact interval with $\left[\lambda_{1}\left(a^{0}\right), \lambda_{1}\left(a_{0}\right)\right] \cap \Lambda=\emptyset$. Then there exists $a$ number $\delta_{1}>0$ with the property

$$
\Phi_{\lambda}(u) \neq 0, \quad \forall u \in Y: 0<\|u\| \leq \delta_{1}, \forall \lambda \in \Lambda
$$

Proof. Suppose to the contrary that there exist sequences $\left\{\mu_{n}\right\}$ in $\Lambda$ and $\left\{u_{n}\right\}$ in $Y: \mu_{n} \rightarrow \mu^{*} \in$ $\Lambda,\left\|u_{n}\right\|>0$ and $u_{n} \rightarrow 0(n \rightarrow \infty)$ in $Y$, such that $\Phi_{\mu_{n}}\left(u_{n}\right)=0$ for all $n \in \mathbb{N}$. By Lemma 3.2, $u_{n}(t) \geq 0$ for $t \in\left[0, \sigma^{2}(T)\right]_{\mathbb{T}}$.

Set $v_{n}=u_{n} /\left\|u_{n}\right\|$. Then from $L u_{n}=\mu_{n} N\left(u_{n}\right)$, we have $L v_{n}=\mu_{n}\left\|u_{n}\right\|^{-1} N\left(u_{n}\right)$. Since $\left\|u_{n}\right\|^{-1} N\left(u_{n}\right)$ is bounded in $X$, we infer that $\left\{v_{n}\right\}$ is relatively compact in $Y$, hence (for a subsequence) $v_{n} \rightarrow \bar{v}(n \rightarrow \infty)$ with $\bar{v} \geq 0$ in $Y,\|\bar{v}\|=1$. Let $I^{\sigma}: \mathrm{C}_{\mathrm{rd}}\left[0, \sigma^{2}(T)\right]_{\mathbb{T}} \rightarrow$ $\mathrm{C}_{\mathrm{rd}}[0, \sigma(T)]_{\mathbb{T}}$ be defined as $I^{\sigma}(y)=y^{\sigma}$. Then $I^{\sigma}$ is linear. For $u \in Y,\left\|I^{\sigma} u\right\|_{0}=$ $\sup _{t \in[0, \sigma(T)]_{\mathbb{T}}}\left|u^{\sigma}(t)\right|=\|u\|_{0}$. 
Now, from condition (A1), we have that

$$
a_{0}(t) u_{n}^{\sigma}(t)-\xi_{1}\left(t, u_{n}^{\sigma}(t)\right) \leq f\left(t, u_{n}^{\sigma}(t)\right) \leq a^{0}(t) u_{n}^{\sigma}(t)+\xi_{2}\left(t, u_{n}^{\sigma}(t)\right)
$$

According to

$$
\frac{u_{n}^{\sigma}(t)}{\left\|u_{n}\right\|}=\frac{I^{\sigma}\left(u_{n}\right)}{\left\|u_{n}\right\|}=I^{\sigma}\left(\frac{u_{n}}{\left\|u_{n}\right\|}\right)=I^{\sigma}\left(v_{n}\right)=v_{n}^{\sigma},
$$

we get

$$
\mu_{n}\left(a_{0}(t) v_{n}^{\sigma}(t)-\frac{\xi_{1}\left(t, u_{n}^{\sigma}(t)\right)}{\left\|u_{n}\right\|}\right) \leq \mu_{n} \frac{f\left(t, u_{n}^{\sigma}(t)\right)}{\left\|u_{n}\right\|} \leq \mu_{n}\left(a^{0}(t) v_{n}^{\sigma}(t)+\frac{\xi_{2}\left(t, u_{n}^{\sigma}(t)\right)}{\left\|u_{n}\right\|}\right)
$$

Let $\varphi^{0}$ and $\varphi_{0}$ denote the eigenfunctions corresponding to $\lambda_{1}\left(a^{0}\right)$ and $\lambda_{1}\left(a_{0}\right)$, respectively. Denote $\bar{\xi}_{1}(y):=\max _{(t, s) \in[0, \sigma(T)]_{\mathbb{T}} \times[0, y]}\left|\xi_{1}(t, s)\right|$. Then $\bar{\xi}_{1}(y)$ is nondecreasing. From $\lim _{u \rightarrow 0^{+}}\left(\xi_{1}(t, u) / u\right)=0$ uniformly for $t \in[0, \sigma(T)]_{\mathbb{T}}$, we have

$$
\lim _{y \rightarrow 0^{+}} \frac{\bar{\xi}_{1}(y)}{y}=0
$$

According to $L u_{n}=\mu_{n} \bar{f}\left(t, u_{n}^{\sigma}(t)\right)$ and $u_{n}(t) \geq 0$, we have from the first inequality in (3.24) that

$$
\int_{0}^{\sigma(T)}\left[\mu_{n}\left(a_{0}(t) v_{n}^{\sigma}(t)-\frac{\xi_{1}\left(t, u_{n}^{\sigma}(t)\right)}{\left\|u_{n}\right\|}\right)\right] \varphi_{0}^{\sigma}(t) \Delta t \leq \int_{0}^{\sigma(T)} \frac{L u_{n}(t)}{\left\|u_{n}\right\|} \varphi_{0}^{\sigma}(t) \Delta t=\int_{0}^{\sigma(T)} L v_{n}(t) \varphi_{0}^{\sigma}(t) \Delta t .
$$

Notice that

$$
\frac{\xi_{1}\left(t, u_{n}^{\sigma}(t)\right)}{\left\|u_{n}\right\|} \leq \frac{\bar{\xi}_{1}\left(\left\|I^{\sigma} u_{n}\right\|_{0}\right)}{\max \{1,1 / \sigma(T)\}\left\|u_{n}\right\|_{0}} \leq \frac{\bar{\xi}_{1}\left(\left\|I^{\sigma} u_{n}\right\|_{0}\right)}{\left\|I^{\sigma} u_{n}\right\|_{0}} \longrightarrow 0 \quad(n \longrightarrow \infty)
$$

by Lemma 3.1. Let $n \rightarrow \infty$, by integration by parts (2.5), we have

$$
\begin{aligned}
\int_{0}^{\sigma^{2}(T)} \mu^{*} a_{0}(t) \bar{v}^{\sigma}(t) \varphi_{0}^{\sigma}(t) \Delta t & \leq \int_{0}^{\sigma^{2}(T)} L \bar{v}(t) \varphi_{0}^{\sigma}(t) \Delta t \\
& =\int_{0}^{\sigma^{2}(T)} L \varphi_{0}(t) \bar{v}^{\sigma}(t) \Delta t \\
& =\int_{0}^{\sigma^{2}(T)} \lambda_{1}\left(a_{0}\right) a_{0}(t) \varphi_{0}^{\sigma}(t) \bar{v}^{\sigma}(t) \Delta t
\end{aligned}
$$


and consequently

$$
\mu^{*} \leq \lambda_{1}\left(a_{0}\right)
$$

Similarly, we deduce from the second inequality in (3.24) that

$$
\lambda_{1}\left(a^{0}\right) \leq \mu^{*}
$$

Thus, $\lambda_{1}\left(a^{0}\right) \leq \mu^{*} \leq \lambda_{1}\left(a_{0}\right)$. This contradicts $\mu^{*} \in \Lambda$.

Remark 3.4. If $\sigma(T)<\sigma^{2}(T)$, then the value of $\sigma^{2}(T)$ do not contribute to the value of (3.28). Thus we can discuss the integral from 0 to $\sigma^{2}(T)$. For $L \bar{v}$ and $L \varphi_{0}$ are not defined at $\sigma(T)$, we may define the values of $(L \bar{v}) \varphi_{0}^{\sigma}$ and $\left(L \varphi_{0}\right) \bar{v}^{\sigma}$ to be zero at $\sigma(T)$, since the functions $\varphi_{0}^{\sigma}$ and $\bar{v}^{\sigma}$ are zero at $\sigma(T)$. The details of the discussion can be found in [9, Page 497].

Corollary 3.5. For $\lambda \in\left(0, \lambda_{1}\left(a^{0}\right)\right)$ and $\delta \in\left(0, \delta_{1}\right), \operatorname{deg}\left(\Phi_{\lambda}, B_{\delta}, 0\right)=1$.

Proof. Lemma 3.3, applied to the interval $\Lambda=[0, \lambda]$, guarantees the existence of $\delta_{1}>0$ such that for $\delta \in\left(0, \delta_{1}\right)$

$$
u-\tau \lambda L^{-1} N(u) \neq 0, \quad \forall u \in Y: 0<\|u\| \leq \delta, \tau \in[0,1] .
$$

Hence, for any $\delta \in\left(0, \delta_{1}\right)$,

$$
\operatorname{deg}\left(\Phi_{\curlywedge}, B_{\delta}, 0\right)=\operatorname{deg}\left(I, B_{\delta}, 0\right)=1,
$$

which implies the assertion.

On the other hand, we have the following.

Lemma 3.6. Suppose $\lambda>\lambda_{1}\left(a_{0}\right)$. Then there exists $\delta_{2}>0$ such that for all $u \in Y$ with $0<\|u\| \leq \delta_{2}$, for all $\tau \geq 0$,

$$
\Phi_{\lambda}(u) \neq \tau \varphi_{0}
$$

where $\varphi_{0}$ is the positive eigenfunction corresponding to $\lambda_{1}\left(a_{0}\right)$.

Proof. We assume again to the contrary that there exist $\tau_{n} \geq 0$ and a sequence $\left\{u_{n}\right\}$ with $\left\|u_{n}\right\|>0$ and $u_{n} \rightarrow 0$ in $Y$ such that $\Phi_{\lambda}\left(u_{n}\right)=\tau_{n} \varphi_{0}$ for all $n \in \mathbb{N}$. As

$$
L u_{n}=\lambda N\left(u_{n}\right)+\tau_{n} \lambda_{1}\left(a_{0}\right) a_{0}(t) \varphi_{0}^{\sigma}(t)
$$

and $\tau_{n} \mathcal{\lambda}_{1}\left(a_{0}\right) a_{0}(t) \varphi_{0}^{\sigma}(t) \geq 0$ in $[0, T]_{\mathbb{T}}$, we can conclude from Lemma 3.2 that $u_{n}(t) \geq 0$ for $t \in\left[0, \sigma^{2}(T)\right]_{\mathbb{T}}$. 
Notice that $u_{n} \in D(L)$ has a unique orthogonal decomposition

$$
u_{n}=w_{n}+s_{n} \varphi_{0}
$$

with $s_{n} \in \mathbb{R}$. Since $u_{n} \geq 0$ on $\left[0, \sigma^{2}(T)\right]_{\mathbb{T}}$ and $\left\|u_{n}\right\|>0$, we have from (3.35) that $s_{n}>0$.

Choose $\sigma>0$ such that

$$
\sigma<\frac{\lambda-\lambda_{1}\left(a_{0}\right)}{\lambda}
$$

By (A1), there exists $r_{4}>0$, such that

$$
f(t, u) \geq(1-\sigma) a_{0}(t) u, \quad \forall(t, u) \in[0, \sigma(T)]_{\mathbb{T}} \times\left[0, r_{4}\right]
$$

Since $\left\|u_{n}\right\| \rightarrow 0$, there exists $N^{*}>0$, such that

$$
0 \leq u_{n} \leq r_{4}, \quad \forall n \geq N^{*},
$$

and consequently

$$
f\left(t, u_{n}^{\sigma}(t)\right) \geq(1-\sigma) a_{0}(t) u_{n}^{\sigma}(t), \quad \forall n \geq N^{*} .
$$

Applying (3.35) and (3.39), it follows that

$$
\begin{aligned}
s_{n} \lambda_{1}\left(a_{0}\right) \int_{0}^{\sigma^{2}(T)} a_{0}(t)\left[\varphi_{0}^{\sigma}(t)\right]^{2} \Delta t & =\int_{0}^{\sigma^{2}(T)}\left[w_{n}^{\sigma}(t)+s_{n} \phi_{0}^{\sigma}(t)\right] L \varphi_{0}(t) \Delta t \\
& =\int_{0}^{\sigma^{2}(T)} u_{n}^{\sigma}(t) L \varphi_{0}(t) \Delta t \\
& =\int_{0}^{\sigma^{2}(T)} \varphi_{0}^{\sigma}(t) L u_{n}(t) \Delta t \\
& =\lambda \int_{0}^{\sigma^{2}(T)} N\left(u_{n}\right) \varphi_{0}^{\sigma}(t) \Delta t+\tau_{n} \lambda_{1}\left(a_{0}\right) \int_{0}^{\sigma^{2}(T)} a_{0}(t)\left[\varphi_{0}^{\sigma}(t)\right]^{2} \Delta t \\
& \geq \lambda \int_{0}^{\sigma^{2}(T)} N\left(u_{n}\right) \varphi_{0}^{\sigma}(t) \Delta t \\
& \geq \lambda(1-\sigma) \int_{0}^{\sigma^{2}(T)} a_{0}(t) u_{n}^{\sigma}(t) \varphi_{0}^{\sigma}(t) \Delta t \\
& =\lambda(1-\sigma) s_{n} \int_{0}^{\sigma^{2}(T)} a_{0}(t)\left[\varphi_{0}^{\sigma}(t)\right]^{2} \Delta t
\end{aligned}
$$


Thus,

$$
\lambda_{1}\left(a_{0}\right) \geq \lambda(1-\sigma)
$$

This contradicts (3.36).

Corollary 3.7. For $\lambda>\lambda_{1}\left(a_{0}\right)$ and $\delta \in\left(0, \delta_{2}\right), \operatorname{deg}\left(\Phi_{\lambda}, B_{\delta}, 0\right)=0$.

Proof. Let $0<\epsilon \leq \delta_{2}$, where $\delta_{2}$ is the number asserted in Lemma 3.6. As $\Phi_{\curlywedge}$ is bounded in $\bar{B}_{\epsilon}$, there exists $c>0$ such that $\Phi_{\lambda}(u) \neq c \varphi_{0}$, for all $u \in \bar{B}_{\epsilon}$. By Lemma 3.6,

$$
\Phi_{\curlywedge}(u) \neq t c \varphi_{0}, \quad u \in \partial B_{\epsilon}, t \in[0,1]
$$

Hence,

$$
\operatorname{deg}\left(\Phi_{\lambda}, B_{\epsilon}, 0\right)=\operatorname{deg}\left(\Phi_{\lambda}-c \varphi_{0}, B_{\epsilon}, 0\right)=0
$$

Now, using Theorem A, we may prove the following.

Lemma 3.8. $\left[\lambda_{1}\left(a^{0}\right), \lambda_{1}\left(a_{0}\right)\right]$ is a bifurcation interval from the trivial solution for (3.19). There exists an unbounded component $\mathcal{C}$ of positive solutions of (3.19) which meets $\left[\lambda_{1}\left(a^{0}\right), \lambda_{1}\left(a_{0}\right)\right] \times\{0\}$. Moreover,

$$
\mathcal{C} \cap\left[\left(\mathbb{R} \backslash\left[\lambda_{1}\left(a^{0}\right), \lambda_{1}\left(a_{0}\right)\right]\right) \times\{0\}\right]=\emptyset .
$$

Proof. For fixed $n \in \mathbb{N}$ with $\lambda_{1}\left(a^{0}\right)-1 / n>0$, set $\left.a_{n}=\lambda_{1}\left(a^{0}\right)-1 / n<\lambda_{1}\left(a^{0}\right), b_{n}=\lambda_{1}\left(a_{0}\right)+1 / n\right\rangle$ $\lambda_{1}\left(a_{0}\right)$ and $\widehat{\delta}=\min \left\{\delta_{1}, \delta_{2}\right\}$. It is easy to check that for $0<\delta<\widehat{\delta}$, all of the conditions of Theorem A are satisfied. So there exists a connected component $\mathcal{C}_{n}$ of solutions of (3.19) containing $\left[a_{n}, b_{n}\right] \times\{0\}$, and either

(i) $\mathcal{C}_{n}$ is unbounded, or

(ii) $\mathcal{C}_{n} \cap\left[\left(\mathbb{R} \backslash\left[a_{n}, b_{n}\right]\right) \times\{0\}\right] \neq \emptyset$.

By Lemma 3.3, the case (ii) cannot occur. Thus, $\mathcal{C}_{n}$ is unbounded bifurcated from $\left[a_{n}, b_{n}\right] \times\{0\}$ in $\mathbb{R} \times Y$. Furthermore, we have also from Lemma 3.3 that for any closed interval $I \subset\left[a_{n}, b_{n}\right] \backslash$ $\left[\lambda_{1}\left(a^{0}\right), \lambda_{1}\left(a_{0}\right)\right]$, if $u \in\left\{y \in Y \mid(\lambda, y) \in \mathcal{C}_{n}, \lambda \in I\right\}$, then the fact that $\|u\| \rightarrow 0$ in $Y$ is impossible. So $\mathcal{C}_{n}$ must be bifurcated from $\left[\lambda_{1}\left(a^{0}\right), \lambda_{1}\left(a_{0}\right)\right] \times\{0\}$ in $\mathbb{R} \times Y$.

\section{The Main Result}

We obtain the following main result in this paper.

Theorem 4.1. Let (A1), (A2), and (A3) hold. Assuming that either

$$
\lambda_{1}\left(b_{\infty}\right)<1<\lambda_{1}\left(a^{0}\right)
$$


or

$$
\lambda_{1}\left(a_{0}\right)<1<\lambda_{1}\left(b^{\infty}\right)
$$

Then problem (1.1) has at least one positive solution.

Proof. It is clear that any solution to (3.19) of the form $(1, u)$ yields a solution $u$ of problem (1.1). We will show that $\mathcal{C}$ crosses the hyperplane $\{1\} \times Y$ in $\mathbb{R} \times Y$. To do this, it is enough to show that $\mathcal{C}$ joins $\left[\lambda_{1}\left(a^{0}\right), \lambda_{1}\left(a_{0}\right)\right] \times\{0\}$ to $\left[\lambda_{1}\left(b^{\infty}\right), \lambda_{1}\left(b_{\infty}\right)\right] \times\{\infty\}$. Let $\left(\eta_{n}, y_{n}\right) \in \mathcal{C}$ satisfy

$$
\eta_{n}+\left\|y_{n}\right\| \longrightarrow \infty
$$

We note that $\eta_{n}>0$ for all $n \in \mathbb{N}$ since $(0,0)$ is the only solution to (3.19) for $\lambda=0$, and $\mathcal{C} \cap(\{0\} \times Y)=\emptyset$.

Case $1\left(\lambda_{1}\left(b_{\infty}\right)<1<\lambda_{1}\left(a^{0}\right)\right)$. In this case, we show that the interval

$$
\left(\lambda_{1}\left(b_{\infty}\right), \lambda_{1}\left(a^{0}\right)\right) \subseteq\{\lambda \in \mathbb{R} \mid(\lambda, u) \in \mathcal{C}\}
$$

We divide the proof into two steps.

Step 1. We show that $\left\{\eta_{n}\right\}$ is bounded.

Since $\left(\eta_{n}, y_{n}\right) \in \mathcal{C}, L y_{n}=\eta_{n} f\left(t, y_{n}^{\sigma}(t)\right)$. From (A3), we have

$$
L y_{n} \geq \eta_{n} c(t) y_{n}^{\sigma}(t)
$$

Let $\bar{\varphi}$ denote the nonnegative eigenfunction corresponding to $\lambda_{1}(c)$. From (4.5), by integration by parts formula (2.5), we have

$$
\begin{aligned}
\lambda_{1}(c) \int_{0}^{\sigma^{2}(T)} y_{n}^{\sigma}(t) c(t) \bar{\varphi}^{\sigma}(t) \Delta t & =\int_{0}^{\sigma^{2}(T)}(L \bar{\varphi})(t) y_{n}^{\sigma}(t) \Delta t \\
& =\int_{0}^{\sigma^{2}(T)}\left(L y_{n}\right)(t) \bar{\varphi}^{\sigma}(t) \Delta t \\
& \geq \eta_{n} \int_{0}^{\sigma^{2}(T)} c(t) y_{n}^{\sigma}(t) \bar{\varphi}^{\sigma}(t) \Delta t .
\end{aligned}
$$

Thus,

$$
\eta_{n} \leq \lambda_{1}(c)
$$

Step 2. We show that $\mathcal{C}$ joins $\left[\lambda_{1}\left(a^{0}\right), \lambda_{1}\left(a_{0}\right)\right] \times\{0\}$ to $\left[\lambda_{1}\left(b^{\infty}\right), \lambda_{1}\left(b_{\infty}\right)\right] \times\{\infty\}$. 
From (4.3) and (4.7), we have that $\left\|y_{n}\right\| \rightarrow \infty$. Notice that (3.19) is equivalent to the equation

$$
y_{n}(t)=\eta_{n} \int_{0}^{\sigma^{2}(T)} H(t, s) f\left(s, y_{n}^{\sigma}(s)\right) \Delta s
$$

where $H(t, s)$ is the Green's function for problem $-u^{\Delta \Delta}=0, u(0)=u\left(\sigma^{2}(T)\right)=0$. So we have from (A1),

$$
\begin{aligned}
& \eta_{n} \int_{0}^{\sigma^{2}(T)} H(t, s)\left[b^{\infty}(s) y_{n}^{\sigma}(s)+\zeta_{2}\left(s, y_{n}^{\sigma}(s)\right)\right] \Delta s \\
& \quad \geq y_{n}(t) \\
& \quad \geq \eta_{n} \int_{0}^{\sigma^{2}(T)} H(t, s)\left[b_{\infty}(s) y_{n}^{\sigma}(s)+\zeta_{1}\left(s, y_{n}^{\sigma}(s)\right)\right] \Delta s .
\end{aligned}
$$

We divide the both sides of (4.9) by $\left\|y_{n}\right\|$ and set $v_{n}=y_{n} /\left\|y_{n}\right\|$. Since $v_{n}$ is bounded in $Y$, choosing a subsequence and relabelling if necessary, we see that $v_{n} \rightarrow \widetilde{v}(n \rightarrow \infty)$ for some $\tilde{v} \in Y$ with $\tilde{v} \geq 0$ in $Y,\|\tilde{v}\|=1$ and $\eta_{n} \rightarrow \eta^{*}(n \rightarrow \infty)$. Similar to the proof of Lemma 3.3, we have that

$$
\lim _{n \rightarrow \infty} \frac{\zeta_{i}\left(s, y_{n}^{\sigma}(s)\right)}{\left\|y_{n}\right\|}=0, \quad i=1,2
$$

and $n \rightarrow \infty$, it is easy to verify that

$$
\eta^{*} \int_{0}^{\sigma^{2}(T)} H(t, s) b^{\infty}(s) \widetilde{v}^{\sigma}(s) \Delta s \geq \widetilde{v}(t) \geq \eta^{*} \int_{0}^{\sigma^{2}(T)} H(t, s) b_{\infty}(s) \widetilde{v}^{\sigma}(s) \Delta s,
$$

which implies that

$$
\eta^{*} b_{\infty}(t) \widetilde{v}^{\sigma}(t) \leq L \tilde{v} \leq \eta^{*} b^{\infty}(t) \widetilde{v}^{\sigma}(t)
$$

Let $\varphi^{\infty}$ and $\varphi_{\infty}$ denote the nonnegative eigenfunctions corresponding to $\lambda_{1}\left(b^{\infty}\right)$ and $\lambda_{1}\left(b_{\infty}\right)$, respectively. Then we have from the first inequality in (4.12) that

$$
\int_{0}^{\sigma^{2}(T)} \eta^{*} b_{\infty}(t) \widetilde{v}^{\sigma}(t) \varphi_{\infty}^{\sigma}(t) \Delta t \leq \int_{0}^{\sigma^{2}(T)} L \widetilde{v}(t) \varphi_{\infty}^{\sigma}(t) \Delta t
$$


By integration by parts formula (2.5), we obtain that

$$
\begin{aligned}
\eta^{*} \int_{0}^{\sigma^{2}(T)} b_{\infty}(t) \varphi_{\infty}^{\sigma}(t) \tilde{v}^{\sigma}(t) \Delta t & \leq \int_{0}^{\sigma^{2}(T)} L \varphi_{\infty}(t) \widetilde{v}^{\sigma}(t) \Delta t \\
& =\lambda_{1}\left(b_{\infty}\right) \int_{0}^{\sigma^{2}(T)} b_{\infty}(t) \varphi_{\infty}^{\sigma}(t) \widetilde{v}^{\sigma}(t) \Delta t
\end{aligned}
$$

and consequently

$$
\eta^{*} \leq \lambda_{1}\left(b_{\infty}\right)
$$

Similarly, we deduce from the second inequality in (4.12) that

$$
\lambda_{1}\left(b^{\infty}\right) \leq \eta^{*}
$$

Thus,

$$
\lambda_{1}\left(b^{\infty}\right) \leq \eta^{*} \leq \lambda_{1}\left(b_{\infty}\right)
$$

So $C$ joins $\left[\lambda_{1}\left(a^{0}\right), \lambda_{1}\left(a_{0}\right)\right] \times\{0\}$ to $\left[\lambda_{1}\left(b^{\infty}\right), \lambda_{1}\left(b_{\infty}\right)\right] \times\{\infty\}$.

Case $2\left(\lambda_{1}\left(a_{0}\right)<1<\lambda_{1}\left(b^{\infty}\right)\right)$. In this case, if $\left(\eta_{n}, y_{n}\right) \in \mathcal{C}$ is such that

$$
\begin{gathered}
\lim _{n \rightarrow \infty}\left(\eta_{n}+y_{n}\right)=\infty, \\
\lim _{n \rightarrow \infty} \eta_{n}=\infty,
\end{gathered}
$$

then

$$
\left(\lambda_{1}\left(a_{0}\right), \lambda_{1}\left(b^{\infty}\right)\right) \subseteq\{\lambda \in(0, \infty) \mid(\lambda, u) \in \mathcal{C}\}
$$

and, moreover,

$$
(\{1\} \times Y) \cap \mathcal{C} \neq \emptyset
$$

Assuming that $\left\{\eta_{n}\right\}$ is bounded, applying a similar argument to that used in Step 2 of Case 1, after taking a subsequence and relabelling if necessary, it follows that

$$
\eta_{n} \longrightarrow \eta^{*} \in\left[\lambda_{1}\left(a_{0}\right), \lambda_{1}\left(b^{\infty}\right)\right], \quad y_{n} \longrightarrow \infty, \quad \text { as } n \longrightarrow \infty
$$

Again $C$ joins $\left[\lambda_{1}\left(a^{0}\right), \lambda_{1}\left(a_{0}\right)\right] \times\{0\}$ to $\left[\lambda_{1}\left(b^{\infty}\right), \lambda_{1}\left(b_{\infty}\right)\right] \times\{\infty\}$ and the result follows. 


\section{Acknowledgments}

This work supported by China Postdoctoral Science Foundation funded Project (no. 201104602 and no. 20100481239), General Project for Scientific Research of Liaoning Educational Committee (no. L2011200), Teaching and Research Project of DUFE (no. YY12012), and the NSFC (no. 71201019).

\section{References}

[1] R. P. Agarwal and D. O'Regan, "Nonlinear boundary value problems on time scales," Nonlinear Analysis, vol. 44, no. 4, pp. 527-535, 2001.

[2] F. M. Atici and G. Sh. Guseinov, “On Green's functions and positive solutions for boundary value problems on time scales," Journal of Computational and Applied Mathematics, vol. 141, no. 1-2, pp. 75-99, 2002.

[3] R. I. Avery and D. R. Anderson, "Existence of three positive solutions to a second-order boundary value problem on a measure chain," Journal of Computational and Applied Mathematics, vol. 141, no. 1-2, pp. 65-73, 2002.

[4] C. J. Chyan and J. Henderson, "Eigenvalue problems for nonlinear differential equations on a measure chain," Journal of Mathematical Analysis and Applications, vol. 245, no. 2, pp. 547-559, 2000.

[5] L. H. Erbe and A. C. Peterson, "Positive solutions for a nonlinear differential equation on a measure chain," Mathematical and Computer Modelling, vol. 32, no. 5-6, pp. 571-585, 2000.

[6] H. Luo and R. Ma, "Nodal solutions to nonlinear eigenvalue problems on time scales," Nonlinear Analysis, vol. 65, no. 4, pp. 773-784, 2006.

[7] R. Ma and J. Xu, "Bifurcation from interval and positive solutions of a nonlinear fourth-order boundary value problem," Nonlinear Analysis, vol. 72, no. 1, pp. 113-122, 2010.

[8] P. H. Rabinowitz, "Some aspects of nonlinear eigenvalue problems," The Rocky Mountain Journal of Mathematics, vol. 3, pp. 161-202, 1973.

[9] F. A. Davidson and B. P. Rynne, "Curves of positive solutions of boundary value problems on timescales," Journal of Mathematical Analysis and Applications, vol. 300, no. 2, pp. 491-504, 2004.

[10] F. A. Davidson and B. P. Rynne, "Global bifurcation on time scales," Journal of Mathematical Analysis and Applications, vol. 267, no. 1, pp. 345-360, 2002. 


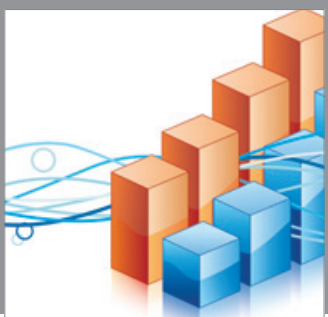

Advances in

Operations Research

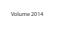

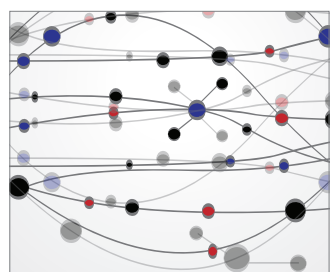

\section{The Scientific} World Journal
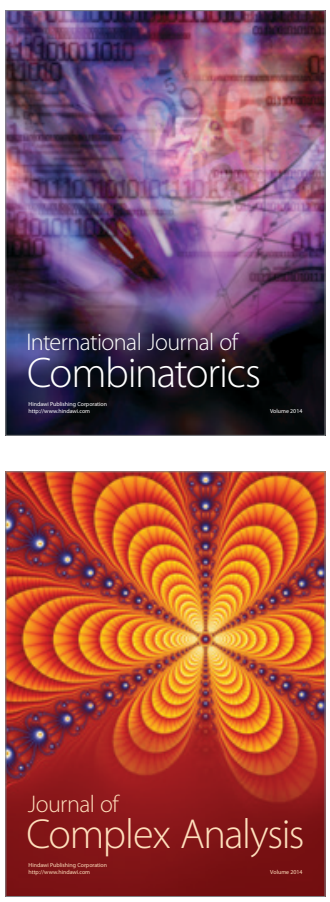

International Journal of

Mathematics and

Mathematical

Sciences
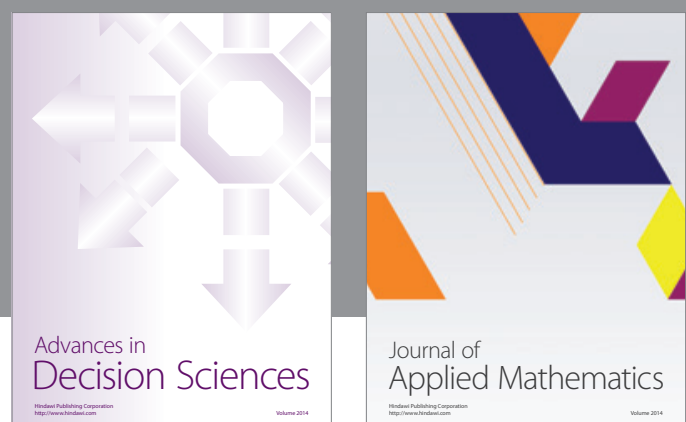

Journal of

Applied Mathematics
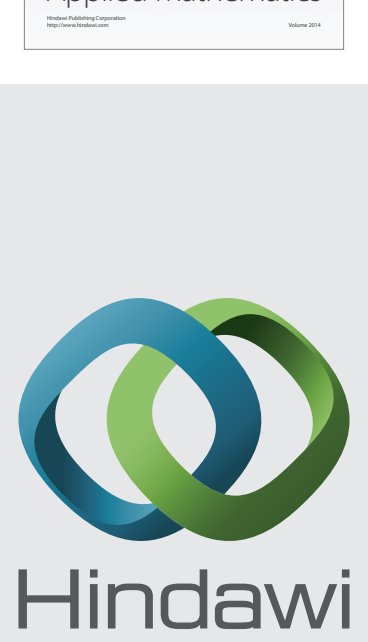

Submit your manuscripts at http://www.hindawi.com
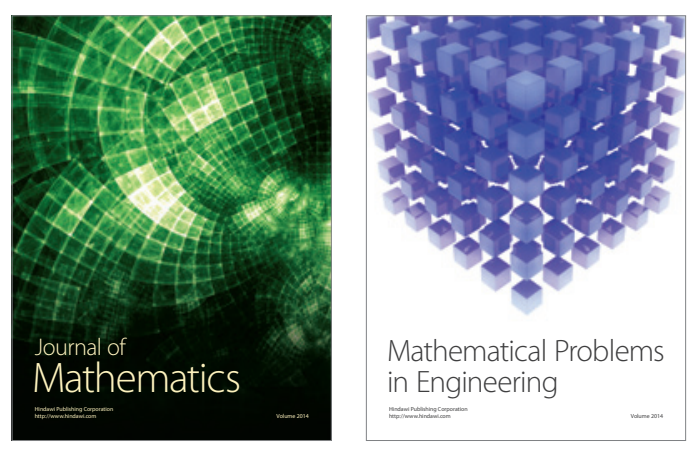

Mathematical Problems in Engineering
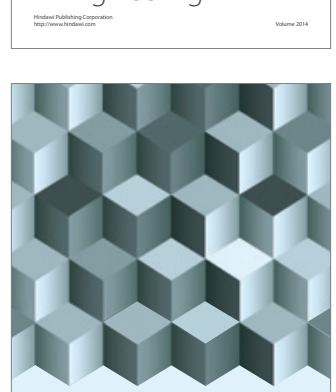

Journal of

Function Spaces
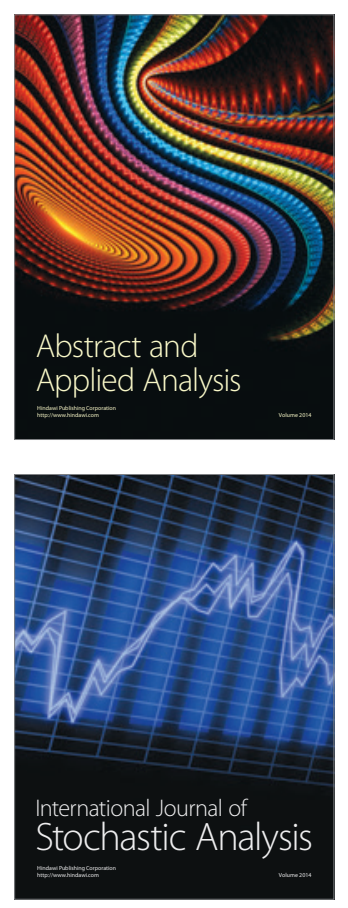

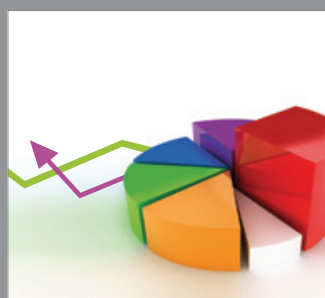

ournal of

Probability and Statistics

Promensencen
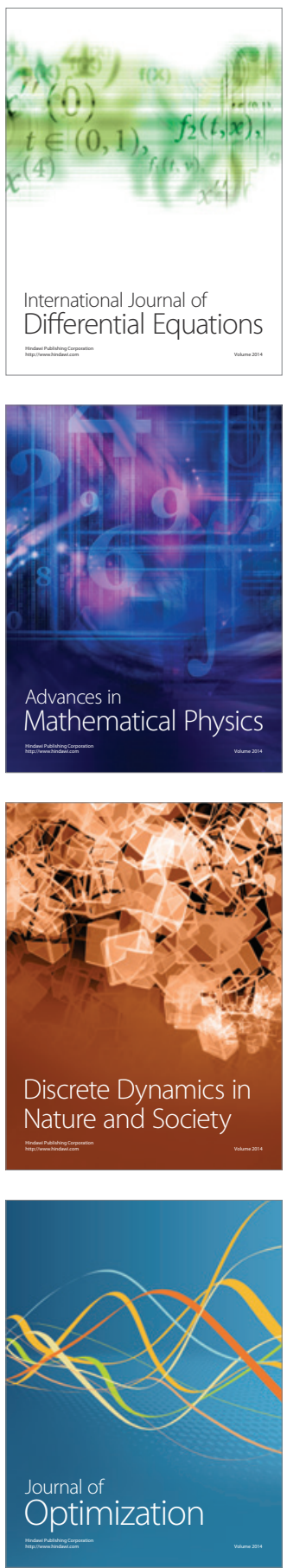